\title{
Prolongation of Corrected QT Interval in Diabetic Patients with Ketoacidosis
}

\author{
Ahmed M, Hussein ${ }^{1}$, Hala Abdel Moniem*1, Ahmed Bendary ${ }^{2}$, Amira K. El-Alfy ${ }^{1}$ \\ Departments ${ }^{1}$ Internal Medicine and ${ }^{2}$ Cardiology, Faculty of Medicine, Benha University, Egypt \\ *Corresponding author: Hala Abdel Moniem, Mobile: (+20)1003295100, E-Mail: halaabdelmonem22@gmail.com
}

\begin{abstract}
Background: Diabetic ketoacidosis (DKA) is the most common acute hyperglycemic complication of diabetes. According to a recent report DKA affects approximately 8 per 1000 diabetics annually. It is associated with significant morbidity and mortality, with a worldwide mortality rate of $2-10 \%$.

Objective: The aim of the work was to assessment of QTc interval prolongation among patients with DKA.

Patients and Methods: This prospective observational cohort study included 100 patients who were diagnosed with DKA. The mean age of patients was $37.29 \pm 11.63$ years, and $53 \%$ of them were males. All patients were subjected to detailed history taking, full clinical examination, laboratory investigations and 12-lead ECG.

Results: Frequency of Prolonged QTmaxc amongst studied patients was 59\%. Mean QTmaxc declined significantly after treatment to be $414.6 \pm 44.1 \mathrm{~ms}$ compared to $482.45 \pm 63.56 \mathrm{~ms}$ before treatment with $\mathrm{p}<0.0001$ and Frequency of prolonged QTmaxc was significantly decreased with treatment from $59 \%$ to $20 \%$. Anion gap was significantly higher for Prolonged QTmaxc patients compared to normal QTmaxc patients $\mathrm{p}<0.0001$. While ABG (PH, HCO3) were significantly lower for Prolonged QTmaxc patients compared to normal QTmaxc patients $\mathrm{p}<0.0001$. Logistic regression revealed that anion gap was significant independent risk factor for QTmaxc prolongation while.

Conclusion: patients with DKA have a potential risk of QTmaxc interval prolongation due to acidosis regardless electrolytes abnormalities, and associated with a relative risk of 1.732-fold for mortality. Carful measuring of anion gap at time of admission can be used in diagnosis and prediction of occurrence of prolonged QTmaxc with high sensitivity and specificity.
\end{abstract}

Keywords: Prolongation, QT Interval, Diabetes mellitus, Ketoacidosis

\section{INTRODUCTION}

Diabetes mellitus is a global healthcare burden because the prevalence of this common disease is increasing significantly. Urbanization and economic trend are the two major factors influencing the prevalence of diabetes, which differs among several population groups. There is a close link between diabetes and cardiovascular diseases, and Electrocardiogram (ECG) is a routine cardiac examination ${ }^{(1)}$. Diabetic ketoacidosis (DKA) is the most common acute hyperglycemic complication of diabetes. According to a recent report DKA affects approximately 8 per 1000 diabetics annually. It is associated with significant morbidity and mortality, with a worldwide mortality rate of $2-10 \%{ }^{(2)}$.

Cardiac arrest has been described as a complication of DKA and is often attributed to electrolyte disturbances such as hyperkalemia or hypomagnesemia. Ventricular arrhythmias including bigeminy and trigeminy have been described and there are multiple reports of sudden death while asleep in patients with diabetes mellitus which have been attributed to possible malignant arrhythmias ${ }^{(3)}$.

QT interval in ECG is measured from the beginning of QRS complex to the end of the T wave, as a measure of 'electrical' ventricular contraction time and is highly dependent on heart rate. Therefore, QT interval length corrected by heart rate (QTc interval) is used in clinical practice. This interval is easily accessible by digital ECG as a simple, low-cost measure to predict all-cause and cardiovascular mortality in emergency and intensive care units ${ }^{(4)}$.

During DKA, ketosis or acidosis may directly affect cardiac repolarization with prolongation of QTc interval, leading to arrhythmia and cardiac arrest ${ }^{(5)}$. Most of the previous studies had reported prolongation of QTc interval in children during DKA with a suggestion of whether ketosis or acidosis may directly affect cardiac repolarization and cause arrhythmia and cardiac arrest during DKA, but few data were reported in adult ${ }^{(5,6)}$.

The aim of the work was to assessment of QTc interval prolongation among patients with DKA.

\section{PATIENTS AND METHODS}

This prospective observational cohort study included a total of 100 patients with DKA, attending at medical ICU, Benha University Hospitals. This study was conducted between March 2020 to February 2021.

\section{Inclusion criteria:}

- Patients admitted to medical ICU with diagnosis of DKA.

- Patients aged $\geq 18$ years old.

- DKA was diagnosed by all of the following criteria: plasma glucose more than $250 \mathrm{mg} / \mathrm{dl}$, arterial $\mathrm{pH}$ of less than or equal to 7.30, 
bicarbonate level of less than or equal to $18 \mathrm{mEq} / \mathrm{l}$, and an anion gap of more than 12 (adjusted for albumin) ${ }^{(7)}$.

\section{Exclusion criteria:}

We tried to eliminate all confounding factors that could cause QTc prolongation or interfere in our measurement of QTc. Patients were ineligible if they were:

- Having structural heart disease (left ventricular hypertrophy, heart failure, and myocardial ischemia which was excluded by history and ECG).

- Patients with hyperthyroidism.

- Patients with hypercholesterolemia.

- Patients with BMI more than $30 \mathrm{~kg} / \mathrm{m}^{2}$.

- Patients who were taking medications known to affect QTc.

- Patients with a wide complex QRS, abnormal ST segment, or T-wave changes.

All patients were subjected to the following:

A. Detailed history taking.

B. Full clinical examination.

C. All patients received treatment according to a standardized DKA protocol ${ }^{(7)}$.

D. The following laboratory investigations: Complete blood picture. Urine analysis. Blood gases. Random plasma sugar. Electrolytes. Kidney function tests. Liver function tests. Lipid profile. Thyroid-stimulating hormone, and HbAlc.

E. Initial anion gap was calculated $[\mathrm{AG}=\mathrm{Na}-(\mathrm{Cl}+$ $\mathrm{HCO} 3)]$.

F. A standard 12-lead ECG was recorded at $25 \mathrm{~mm} / \mathrm{s}$ in the first $6 \mathrm{~h}$ of admission and after the control of DKA episode.

G. QT interval was measured manually from at least 8-leads. At least two consecutive QT intervals were measured in each lead. QTc interval was measured from the onset of the QRS complex to the end of the $\mathrm{U}$ wave or $\mathrm{T}$ wave. The end of the QT interval was defined as the intersection of a tangent to the steepest downslope of the dominant repolarization wave with the isoelectric line. When $\mathrm{T}$ and $\mathrm{U}$ waves were fused, the $\mathrm{U}$ component was included (QTU) for measurement purposes, if a discrete $\mathrm{U}$ wave was seen after the $\mathrm{T}$ wave it was excluded from measurement (QTc).

Maximum QT interval in all measured leads (QTmax) and heart rate-corrected QTmax (QTmaxc) were calculated in milliseconds.

QTmaxc $=\mathbf{Q T} \max / \sqrt{\mathbf{R}}-\mathbf{R}$ interval according to Bazett's formula ${ }^{(8)}$, where QTmaxc equal to or above $450 \mathrm{~ms}$ in men or equal to or above $460 \mathrm{~ms}$ in women was considered a sign for prolonged QT interval.

\section{Ethical Consideration:}

Written informed and oral consents were taken from the relatives of patients who participated in the study in addition to the approval for performing the study that was obtained from medical ICU of Internal Medicine Department, Benha University, after taking the Institutional Review Board (IRB) approval. The study was performed according to the recommendations of the Helsinki declaration

\section{Statistical Analysis}

Data were analyzed using IBM SPSS 23.0 for windows (SPSS Inc., Chicago, IL, USA) and NCSS 11 for windows (NCSS LCC., Kaysville, UT, USA). Quantitative data were expressed as mean \pm standard deviation (SD). Qualitative data were expressed as frequency and percentage. Continuous data were checked for normality by using Shapiro Walk test. The following tests were done: Independent-samples t-test of significance was used when comparing between two means. Paired t test was used to compare between paired normally distributed variables. Mann Whitney $\mathrm{U}$ test was used when comparing two means of not normally distributed data. Chi-square $\left(\mathrm{X}^{2}\right)$ test of significance was used in order to compare proportions between two qualitative parameters. Fisher Exact test is a test of significance that is used in the place of chi square test in 2 by 2 tables, especially in cases of small samples. Pearson's correlation coefficient was calculated to assess relationship between various study variables, (+) sign indicate direct correlation \& (-) sign indicate inverse correlation, also values near to 1 indicate strong correlation \& values near 0 indicate weak correlation. The logistic regression was used to describe data and to explain the relationship between one dependent binary variable and one or more nominal, ordinal, interval or ratio-level independent variables. All tests were two sided. $\mathrm{P}$ value $<0.05$ was considered significant.

\section{RESULTS}

Patient ages ranged between 19 and 63 years, with Mean \pm SD was $37.29 \pm 11.63$ years. Regarding sex, $47 \%$ of studied patients were females, and $53 \%$ were males (Table 1).

Table (1): Demographic characters of studied patients.

\begin{tabular}{|c|c|c|}
\hline & Mean \pm SD & Range \\
\hline Age (years) & $37.29 \pm 11.63$ & $19-63$ \\
\hline Sex & n. & $\%$ \\
\hline $\begin{array}{ll}\text { - } & \text { Females } \\
\text { - } & \text { Males }\end{array}$ & $\begin{array}{l}47 \\
53\end{array}$ & $\begin{array}{l}47 \\
53\end{array}$ \\
\hline
\end{tabular}

The mean BMI of studied patients was 22.93 $\left(\mathrm{kg} / \mathrm{m}^{2)}\right.$ ranged between 19.49 to 23.77 and duration of diabetes was ranged between 1-12 years with Mean \pm SD $6.18 \pm 2.89$ year, $24.0 \%$ of the study population 
were smokers. The mean $\mathrm{HbA} 1 \mathrm{C}$ in the studied patients was $10.20 \pm 1.76 \%$ ranged from 7.6-14.5 and RBG mean was $430.26 \pm 100.2$ ranged from $278-617$ $\mathrm{mg} / \mathrm{dl}$. Regarding Lipid profile, means of TGs, TC, LDL, and HDL were $(116.89,164.83,106.06$, and $41.06 \mathrm{mg} / \mathrm{dl}$ ) respectively. Regarding blood picture, mean $\mathrm{Hb}$ level was $12.9 \mathrm{~g} / \mathrm{dl}$ ranged from 5.8 to 16.9 $\mathrm{g} / \mathrm{dl}$, whereas mean WBC was $12.59 \times 10^{3}$ ranged from 4.8 to $32 \times 10^{3}$ and mean Platelet was $236.83 \times 10^{3}$ ranged from $118-423 \times 10^{3}$.

Regarding renal function tests, mean Creatinine, urea, and ACR were $1.96 \pm 9.2,37.37 \pm 4.25$, and $85.86 \pm 146.9$ respectively. Regarding liver function, mean levels of AST and ALT were 36.65 \pm 27.37 , and
$32.44 \pm 33.41$ IU respectively and mean serum Albumin was $4.16 \pm 0.39 \mathrm{gm} / \mathrm{dl}$. Mean thyroid stimulating hormones was $2.81 \pm 1.29$ ranged from 0.8 to $5.4 \mathrm{mIU} / \mathrm{L}$. And finally, regarding blood electrolyte means of $\mathrm{Na}, \mathrm{Ca}, \mathrm{Po} 4, \mathrm{Mg}$, and $\mathrm{K}$ were $142.45 \pm 3.59 \mathrm{mEq} / \mathrm{L}, \quad 9.12 \pm 0.67 \mathrm{mg} / \mathrm{dL}$ $3.61 \pm 0.48 \mathrm{mg} / \mathrm{dL}, \quad 2.38 \pm 0.5 \quad \mathrm{mg} / \mathrm{dL}, \quad$ and $4.61 \pm 0.51 \mathrm{mmol} / \mathrm{L}$ respectively (Table 2 ).

As regard Anion gap of studied patients, the result ranged between 21-37 with mean \pm SD 29.19 \pm 4.53 . $\mathrm{PH}$ was ranged between 6.96-7.23 in the studied patients with Mean \pm SD (7.09 \pm 0.069$)$. HCO3 was ranged between 7-15 in the studied patients with Mean \pm SD 10.45 \pm 1.81 (Table 2).

\section{Table (2): Risk factor and laboratory parameters of studied patients.}

\begin{tabular}{|c|c|c|}
\hline \multicolumn{2}{|r|}{ Parameters } & Mean \pm SD \\
\hline \multicolumn{2}{|c|}{ BMI (kg) } & $22.93 \pm 0.78$ \\
\hline \multicolumn{2}{|c|}{ Duration of diabetes (years) } & $6.18 \pm 2.89$ \\
\hline \multicolumn{2}{|c|}{$\mathrm{HbA1C}(\mathrm{mmol} / \mathrm{l})$} & $10.20 \pm 1.76$ \\
\hline \multicolumn{2}{|c|}{ RBG (mg/dL) } & $430.26 \pm 100.2$ \\
\hline \multicolumn{2}{|c|}{ TGs (ng/mL) } & $116.89 \pm 18.49$ \\
\hline \multicolumn{2}{|c|}{ TC } & $164.83 \pm 18.82$ \\
\hline \multicolumn{2}{|c|}{ LDL (mg/dl) } & $106.06 \pm 17.99$ \\
\hline \multicolumn{2}{|c|}{ HDL (mg/dL) } & $41.06 \pm 8.48$ \\
\hline \multicolumn{2}{|c|}{ Hb (mmol/L) } & $12.9 \pm 2.06$ \\
\hline \multicolumn{2}{|c|}{ WBC $\left(10^{\wedge} 3\right.$ cells/ mm3) } & $12.59 \pm 2.17$ \\
\hline \multicolumn{2}{|c|}{ Platelet $\left(10^{\wedge} 3\right.$ cells $\left./ \mathrm{mm} 3\right)$} & $236.83 \pm 7.29$ \\
\hline \multicolumn{2}{|c|}{ Creatinine (mg/dL) } & $1.96 \pm 0.2$ \\
\hline \multicolumn{2}{|c|}{ Urea $(\mathrm{mmol} / \mathrm{L})$} & $37.37 \pm 4.25$ \\
\hline \multicolumn{2}{|c|}{ ACR (mg/g) } & $85.86 \pm 16.9$ \\
\hline \multicolumn{2}{|c|}{ AST (U/L) } & $36.65 \pm 5.37$ \\
\hline \multicolumn{2}{|c|}{$\operatorname{ALT}(\mathbf{U} / \mathbf{L})$} & $32.44 \pm 3.41$ \\
\hline \multicolumn{2}{|c|}{$\operatorname{Albumin}(g / L)$} & $4.16 \pm 0.39$ \\
\hline \multicolumn{2}{|c|}{ TSH (milliunits/L) } & $2.81 \pm 0.29$ \\
\hline \multicolumn{2}{|c|}{$\mathrm{Na}(\mathrm{mEq} / \mathrm{L})$} & $142.45 \pm 3.59$ \\
\hline \multicolumn{2}{|c|}{$\mathrm{Ca}(\mathrm{mmol} / \mathrm{L})$} & $9.12 \pm 0.67$ \\
\hline \multicolumn{2}{|c|}{ Po4 (mmol/L) } & $3.61 \pm 0.48$ \\
\hline \multicolumn{2}{|c|}{$\mathrm{Mg}(\mathrm{mEq} / \mathrm{L})$} & $2.38 \pm 0.5$ \\
\hline \multicolumn{2}{|l|}{$\mathbf{K}$} & $4.61 \pm 0.51$ \\
\hline \multicolumn{2}{|c|}{ Anion gap } & $29.19 \pm 4.53$ \\
\hline \multirow[t]{2}{*}{ ABG } & PH & $7.09 \pm 0.069$ \\
\hline & $\mathrm{HCO} 3$ & $10.45 \pm 1.81$ \\
\hline
\end{tabular}

Ketoacidosis patients at time of admission ICU 59\% of them had Prolonged QTmaxc value. The Mean \pm SD and range of QTmaxc was decline significantly at post treatment phase to be $414.6 \pm 44.1 \mathrm{~ms}$ with range from 350 to $520 \mathrm{~ms}$ compared to $482.45 \pm 63.56 \mathrm{~ms}$ with range from 380 to $590 \mathrm{~ms}$ at pre-treatment phase $\mathrm{p}=0.0001$. Frequency of prolonged QTmaxc was significantly decreased with treatment from 59\% to $20 \%$ (Table 3 ).

Table (3): Changes of QTmaxc throughout study phases.

\begin{tabular}{|l|c|c|c|c|}
\hline & Pre -treatment & Post- treatment & Paired t & p-value \\
\hline QTmaxc & $482.45 \pm 63.56$ & $414.6 \pm 44.1$ & 17.41 & 0.0001 \\
Mean \pm SD & $380-590$ & $350-520$ & & \\
Range & $59 \%$ & $20 \%$ & $\chi 2=16.45$ & 0.0001 \\
\hline Frequency of prolonged QTmaxc & &
\end{tabular}

$\chi 2$ Chi square test, Paired $t$ test $\mathrm{p}<0.05$ statistically significant

Comparison of the data between the patients with prolonged QTmaxc and patients with no prolonged QTmaxc revealed that, anion gap, was significantly higher for Prolonged QTmaxc patients compared to normal QTmaxc patients 
$\mathrm{p}=0.0001$. While, ABG (PH, HCO3) were significantly lower for Prolonged QTmaxc patients compared to normal QTmaxc patients $\mathrm{p}=0.0001$ (Table 4).

Table (4): Comparison between Prolonged QTmaxc cases and non-prolonged ones regarding anion gap, ABG at the time of admission.

\begin{tabular}{|l|c|c|c|c|c|}
\hline \multicolumn{2}{|c|}{} & $\begin{array}{c}\text { Prolonged QTmaxc } \\
(\mathbf{n . 5 9 )}\end{array}$ & $\begin{array}{c}\text { Normal QTmaxc patients } \\
(\mathbf{n . 4 1 )}\end{array}$ & $\mathbf{t}$ & p-value \\
\hline \multirow{2}{*}{ Anion gap } & $32.34 \pm 2.83$ & $24.65 \pm 1.89$ & 16.254 & 0.0001 \\
\hline \multirow{2}{*}{ ABG } & pH & $7.05 \pm 0.052$ & $7.15 \pm 0.031$ & 12.904 & 0.0001 \\
\cline { 2 - 6 } & HCO3 & $9.21 \pm 1.01$ & $12.24 \pm 1.02$ & 14.678 & 0.0001 \\
\hline
\end{tabular}

(t)test of significant significant $\mathrm{p}<0.05$

There was positive correlation between QTmaxc at the time of admission to ICU and anion gap. While there was negative correlation between QTmaxc at the time of admission to ICU and $\mathrm{pH}, \mathrm{HCO}$. There was statistically insignificant relation between anion gap, $\mathrm{ABG}$ at the time of admission and survival outcome of studied patients $\mathrm{p}>0.05$. While pre and post QTmaxc value was significantly higher for dead patients compared to survival patients $\mathrm{p}=0.0001$, $\mathrm{p}=0.004$ respectively. QTmaxc prolongation carried a relative risk of 1.732 -fold for mortality in patients with diabetic ketoacidosis with $95 \%$ confidence interval 1.461 to 2.054 (Table 5).

Table (5)1: Incidence of in hospital mortality among patients with Prolonged QTmaxc.

\begin{tabular}{|c|c|c|c|c|c|}
\hline & \multirow{2}{*}{$\begin{array}{c}\text { Prolonged } \\
\text { QTmaxc } \\
(\text { n.59) }\end{array}$} & \multirow{2}{*}{$\begin{array}{c}\text { Normal } \\
\text { QTmaxc } \\
(\mathbf{n . 4 1 )}\end{array}$} & \multirow[t]{2}{*}{$\mathbf{R R}$} & \multicolumn{2}{|c|}{ (95\% Confidence Interval) } \\
\hline & & & & Lower & Upper \\
\hline $\begin{array}{cl}\text { Mortality (n. \%) } \\
\text { - } & \text { Dead } \\
\text { - } & \text { Survival }\end{array}$ & $\begin{array}{c}3(5.1) \\
56(94.9)\end{array}$ & $\begin{array}{c}0(0.0) \\
41(100.0)\end{array}$ & 1.732 & 1.461 & 2.054 \\
\hline
\end{tabular}

$\mathrm{f}=$ Fisher exact test $(\mathrm{RR})=$ Relative risk

ROC curve of anion gap was assessed to discriminate prolonged QTmaxc from normal QTmaxc diabetic patients with an area under curve (AUC) 0.996. to discriminate prolonged QTmaxc from normal QTmaxc keto acidosis diabetic patients, the sensitivity and specificity obtained to anion gap value for differentiate prolonged QTmaxc from normal QTmaxc keto acidosis diabetic patients, Cutoff equal or more than 27.5 had $98.3 \%$ sensitivity and $97.6 \%$ specificity, Positive predictive value $98.3 \%$, Negative predictive value $97.6 \%$ and accuracy was $98.0 \%$ (Figure 1).

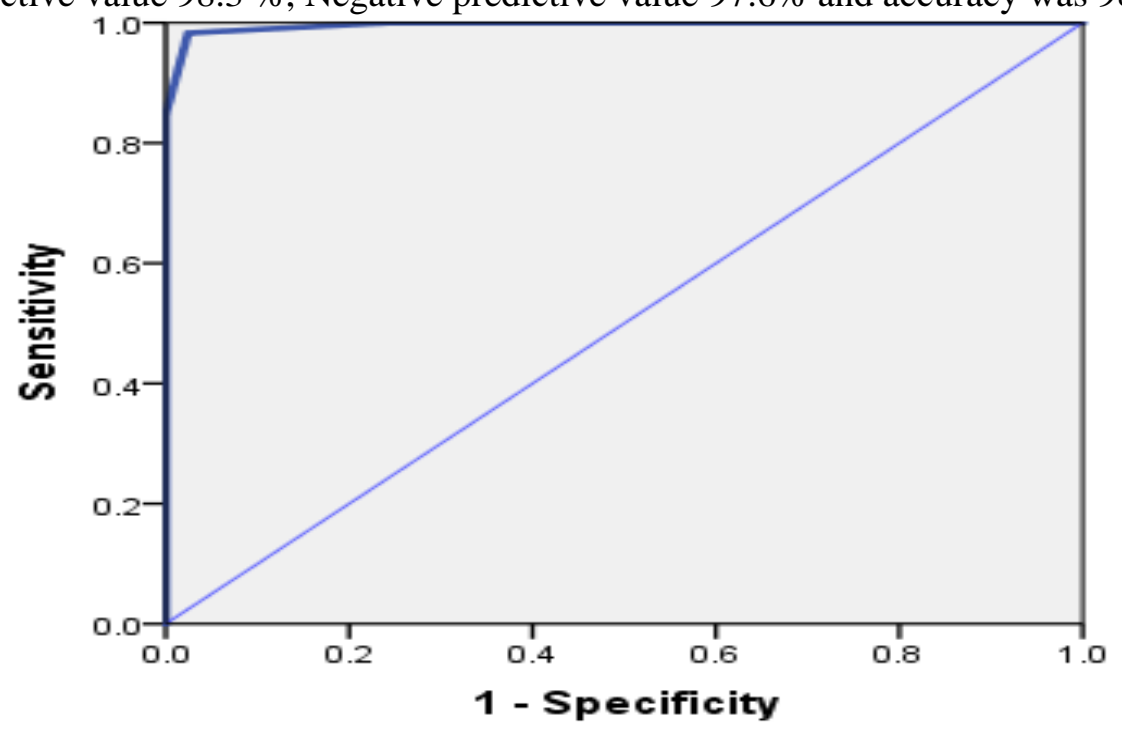

Figure 1: ROC curve of anion gap for prediction of QTmaxc in DKA patients with an area under curve (AUC) 0.996. So, anion gap was very good parameter to discriminate prolonged QTmaxc from normal QTmaxc keto acidosis diabetic patients.

Table (6) shows significant independent risk factor for QTmaxc prolongation, which was anion gap. PH and HCo3 were excluded from model had zero value.

Table (6): Logistic regression for independent predictors of prolonged QTmaxc of ketoacidosis diabetic patients. 


\begin{tabular}{|c|c|c|c|c|c|}
\hline & Wald & Sig. & \multirow{2}{*}{ Odds ratio } & \multicolumn{2}{|c|}{ 95\% C.I. for EXP(B) } \\
\cline { 4 - 5 } & & & & Lower & Upper \\
\hline Anion gap & 7.605 & 0.006 & 80 & 3.5 & 1801 \\
\hline
\end{tabular}

$\mathrm{CI}=95 \%$ confidence interval; odds ratio.

\section{DISCUSSION}

The frequency of QTmaxc interval prolongation among our patients at time of admission to ICU was 59\%. After treatment the prevalence of QTmaxc interval prolongation was significantly diminished $(\mathrm{P}<0.001)$ as only 20 patients still had prolonged QTmaxc. This was supported by Khalil et al. ${ }^{(5)}$, study who reported the frequency of QTmaxc interval prolongation without electrolyte imbalance was seen in 46 (63.9\%) patients. The QTmaxc prolongation was significantly decreased $(\mathrm{P}<0.001)$ with the recovery from DKA in 35 patients; meanwhile, 11 patients had persistent prolonged QTmaxc even after recovery from DKA, which had returned to normal after one week of hospital discharge.

Furthermore, in Kuppermann et al. (9), study, they demonstrate that QTc prolongation was present in almost half of children with DKA. After recovery from the DKA episode, QTc was documented to be normal in all but 2 patients. Also these findings were supported by Aygün et al. ${ }^{(10)}$, who demonstrated that the QTc was found prolonged in 15 of 40 patients and after treatment, the QTc interval continued to be above $450 \mathrm{~ms}$ in only four patients $(10 \%)$

In the present study Mean \pm SD and range of QTmaxc was decline significantly at post treatment phase to be $414.6 \pm 44.1 \mathrm{~ms}$ with range from 350 to $520 \mathrm{~ms}$ compared to $482.45 \pm 63.56 \mathrm{~ms}$ with range from 380 to $590 \mathrm{~ms}$ at pre-treatment phase $\mathrm{p}=0.0001$. Youssef and Farid (11), identified QTc was found to be prolonged (more than $450 \mathrm{~ms}$ ) in 16 patients: 9 patients with new onset diabetes and 7 patients with known diabetes during DKA (range 451-539 ms). The mean QTc values of patients during DKA were $450 \pm 89 \mathrm{~ms}$, which were significantly decreased after recovery from DKA (428 $2 \pm 5 \mathrm{~ms}, \mathrm{P}<0.001)$. Only one child had persistent prolonged QTc $(453 \mathrm{~ms})$ even after recovery from DKA which returned to normal 5 days after hospital discharge.

In accordance with our results Khalil et al. ${ }^{(5)}$, revealed that Patients with prolonged QTmaxc in our study had a significantly lower $\mathrm{pH}$ at presentation compared with those with nonprolonged QTmaxc values, with a significant positive correlation between QTmaxc and Anion gap, and a significant negative correlation between QTmaxc and $\mathrm{pH}$. The QTmaxc interval prolongation was significantly decreased $(\mathrm{P}<0.001)$ with the recovery from DKA in 35 patients; meanwhile, 11 patients had persistent prolonged QTmaxc even after recovery from DKA, which had returned to normal after one week of hospital discharge. This illustrated the effect of acidosis that may precipitate arrhythmias such as reentry, pulsus alternans, and early/delayed afterdepolarization, embracing the findings of other studies $(5,9,11)$.

Also, our findings were supported by Youssef and Farid ${ }^{(11)}$, who revealed that During DKA, patients with prolonged QTc and those with prolonged QTd had a significant higher anion gap and lower $\mathrm{PH}$ at presentation compared to those with normal QTc and QTd values, while no significant difference was found between both groups of patients in other biochemical or clinical data. The initial anion gap was positively correlated with QTc values $(r=0.67, \mathrm{P}<0.0001)$ and QTd value $(r=0.69, P<0.001)$ during DKA. None of children experienced hypoxia or hypoglycemia (blood glucose level $<70 \mathrm{mg} / \mathrm{dL}$ ), 5 patients had mild hypokalemia at the time of ECG recording (range from 3.1 to $3.3 \mathrm{mEq} / \mathrm{L}$ ), while $\mathrm{S}$. $\mathrm{Mg}++$ and $\mathrm{S}$. Ca were within reference range in all patients with no significant correlation between $\mathrm{S}$. Ca, $\mathrm{K}$, and $\mathrm{Mg}++$ with QTc or QTd was found.

Prior studies have reported an association between the ketogenic diet and QTc prolongation Best et $\boldsymbol{a l} .{ }^{(\mathbf{1 2})}$. The study described a series of patients noted to have prolongation of the QTc interval while following a ketogenic diet. A linear relationship between serum ketones and QTc interval was noted. The authors hypothesized that a starvation-like state may create an environment conducive to conduction abnormalities ${ }^{(12)}$. In DKA, a similar pathophysiology exists where the body is unable to use glucose given insulin deficiency and is shifted into a catabolic state and ketogenesis results as the body uses lipolysis to generate energy. Given this shared pathology, we hypothesize that there may be a similar mechanism of QTc prolongation. The mechanism of QTc prolongation is not clear from the findings of this study and may be related to the setting of acidosis. The clinical risk associated with this acquired QTc prolongation is also unknown. Further studies are required to investigate the pathologic mechanism and clinical significance ${ }^{(\mathbf{1 3})}$.

Furthermore, the recent study of Perez et al. ${ }^{(13)}$, described an association between DKA and prolongation of the QTc interval with a prevalence of $31 \%$. QTc prolongation was associated with increasing DKA severity and anion gap, and the association was not accounted for by serum potassium, calcium, and magnesium abnormalities. The authors identified 5 patients with QTc $>500$, all with moderate or severe DKA, and there were no significant electrolyte derangements noted to account for this degree of QTc prolongation these findings further solidify our study.

Prolongation of QT interval is a serious condition that provides substrate for the development of potentially life-threatening arrhythmias torsade de pointes ${ }^{(14)}$. Previous studies supported the association 
of other ketotic conditions with QTc prolongation and deaths in patients receiving ketogenic diets $(\mathbf{1 2}, \mathbf{1 5})$.

Rana et al. ${ }^{(16)}$, who found prolonged QTd to be the best predictor for cardiac death in patients with DM. Moreover, Psallas et al. ${ }^{(17)}$, found that prolonged QTd interval may predict cardiacmortality in patients with diabetes and suggested that it may be a useful adjuvant index in the evaluation of cardiovascular risk in patients with type 2 diabetes and microalbuminuria.

Logistic regression for independent predictors of prolonged QTmaxc of our patients showed that anion gap was significant independent risk factor for QTmaxc prolongation, while $\mathrm{PH}$ and $\mathrm{HCo} 3$ were excluded from model because they had zero value. In contrast Khalil et al. ${ }^{(5)}$ performed a multivariate logistic regression analysis to confirm that $\mathrm{pH}$ was a significant independent predictor for QTmaxc prolongation, with a cutoff value of 7.03, enforcing the results of AdevaAndany et al. ${ }^{(18)}$.

\section{CONCLUSION}

In conclusion the current study revealed that patients with DKA have a potential risk of QTmaxc interval prolongation due to acidosis regardless electrolytes abnormalities, and associated with a relative risk of 1.732-fold for mortality. Carful measuring of anion gap at time of admission can be used in diagnosis and prediction of occurrence of prolonged QTmaxc with high sensitivity and specificity.

Sources of funding: This research did not receive any specific grant from funding agencies in the public, commercial, or not-for-profit sectors.

Author contribution: Authors contributed equally in the study.

Conflicts of interest: No conflicts of interest.

\section{REFERENCES}

1. Glovaci D, Fan W, Wong N (2019): Epidemiology of diabetes mellitus and cardiovascular disease. Curr Cardiol Rep., 21(4):1-8.

2. Harding J, Pavkov M, Magliano D et al. (2019): Global trends in diabetes complications: a review of current evidence. Diabetologia, 62(1):3-16.

3. Skinner J, Marquis-Nicholson R, Luangpraseuth A et al. (2014): Diabetic dead-in-bed syndrome: a possible link to a cardiac ion channelopathy. Case Rep Med., 14: $1-5$.
4. Arai K, Nakagawa Y, Iwata T et al. (2013): Relationships between QT interval and heart rate variability at rest and the covariates in healthy young adults. Auton Neurosci., 173(1-2):53-7.

5. Khalil O, Samir G, Sadek A (2019): Prolonged QTc interval in adults with diabetic ketoacidosis: is it only electrolyte disturbance? Egypt J Intern Med., 31(2):13639.

6. Jaime-Pérez J, García-Arellano G, Herrera-Garza J et al. (2019): Revisiting the complete blood count and clinical findings at diagnosis of childhood acute lymphoblastic leukemia: 10-year experience at a single center. Hematol Transfus cell Ther., 41(1):57-61.

7. Kitabchi A, Umpierrez G, Miles J et al. (2009): Hyperglycemic crises in adult patients with diabetes. Diabetes Care, 32(7):1335-43.

8. Bazett $H$ (1997): An analysis of the time-relations of electrocardiograms. Ann Noninvasive Electrocardiol., 2:177-194.

9. Kuppermann N, Park J, Glatter K et al. (2008): Prolonged QT interval corrected for heart rate during diabetic ketoacidosis in children. Arch Pediatr Adolesc Med., 162(6):544-9.

10. Aygün D, Aygün F, Nişli $K$ et al. (2017): Electrocardiographic changes in children with diabetic ketoacidosis and ketosis. Turkish Arch Pediatr Pediatr Arşivi., 52(4):194-98.

11. Youssef O, Farid S (2012): QTc and QTd in children with type 1 diabetes mellitus during diabetic ketoacidosis. ISRN Pediatr., 12: 619107.

12. Best T, Franz D, Gilbert D et al. (2000): Cardiac complications in pediatric patients on the ketogenic diet. Neurology, 54(12):2328-30.

13. Perez M, Medar S, Quigley L et al. (2021): QTc prolongation in pediatric patients with diabetic ketoacidosis. J Pediatr., 228:235-9.

14. Roden D (2004): Drug-induced prolongation of the QT interval. N Engl J Med., 350(10):1013-22.

15. Stevens A, Robinson D, Turpin J et al. (2002): Sudden cardiac death of an adolescent during dieting. South Med J., 95(9):1047-50.

16. Rana B, Band M, Ogston S et al. (2002): Relation of QT interval dispersion to the number of different cardiac abnormalities in diabetes mellitus. Am J Cardiol., 90(5):483-7.

17. Psallas M, Tentolouris N, Cokkinos A et al. (2006): QT dispersion: comparison between diabetic and nondiabetic individuals and correlation with cardiac autonomic neuropathy. Hell J Cardiol., 47(5):255-62.

18. Adeva-Andany $M$, Fernández-Fernández $C$, Mouriño-Bayolo D et al. (2017): Sodium bicarbonate therapy in patients with metabolic acidosis. https://www.hindawi.com/journals/tswj/2014/627673/ 